\title{
Mitteilungen der Stiftung Michael
}

Z Epileptol 2014 · 27:74-74

DOI 10.1007/ s10309-014-0364-4

(C) Springer-Verlag Berlin Heidelberg 2014
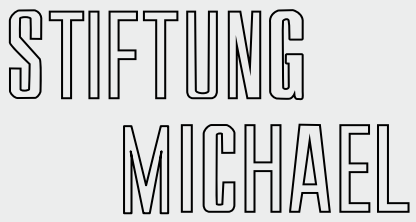

Korrespondenzadresse

STIFTUNG MICHAEL

Alsstraße 12, 53227 Bonn

Tel.: +49-(0)228-94554540

Fax: +49-(0)228-94554542

E-Mail:post@stiftung-michael.de

Homepage: www.stiftung-michael.de

\section{Die Stiftung Michael macht einen weiteren Spendentopf auf}

Der Stiftungsrat hat auf seiner Sitzung am 13.12.2013 beschlossen, sein Stipendienprogramm noch einmal zu erweitern - zur finanziellen Unterstützung von sogenannten „Focused Fellowships“. Dieses Fellowship-Programm orientiert sich am „Department to Department CoOperation-Project“ der European Federation of Neurological Science (http://www.efns.org/ departement-departement-cooperation-programm.1750.html)

Die Höhe eines Stipendiums beträgt Euro 1500,-- plus einem Reisekostenzuschuss.

Die Mindestdauer für eine Hospitation/einen Studienaufenthalt etc. (an ausgewählten Instituten/Abteilungen) beträgt 6 Wochen; längere Aufenthalte sind sicher von Vorteil.

Die Institute/Abteilungen legen fest, welche sprachlichen Voraussetzungen von den $\mathrm{Be}$ werbern erfüllt werden müssen. Es wird auch erwartet, dass die Institute/Abteilungen in einem gewissen Umfang materielle und praktische Hilfestellung geben bei der Unterbringung und Verpflegung der Stipendiaten.

Mit diesem Stipendienprogramm knüpft die Stiftung Michael an ein Stipendienprogramm an, welches in früheren Jahren deutschen, später aber auch einigen ausländischen Ärzten eine Ausbildung in Epileptologie an deutschen EpilepsieZentren ermöglich hat.

Das jetzige Programm bedeutet jedoch eine erhebliche Ausweitung; es will jungen Ärzten und Nachwuchsforschern zunächst aus europäischen Ländern die Möglichkeit geben, an deutschen Zentren für mindestens 6 Wochen zu hospitieren, um klar definierte klinische Prozeduren $-z$. B. fortgeschrittene EEG-Diganostik oder epilepsiebezogene Bildgebung - zu lernen oder an Forschungsprojekten teilzunehmen. Näheres wird in Kürze der Webseite der Stiftung Michael (www.stiftung-michael.de) zu entnehmen sein.

\section{Hinweise auf andere Stipendienprogramme}

Auf die weiteren Stipendienprogramme der Stiftung Michael 1. Ausbildung zur EpilepsieFachassistent (EFA) bzw. Epilepsie-Fachberatung (EFB);

2. VIREPA-Kurse;

3. Praxis-Seminar Gargnano wurde ausführlich in den „Mitteilungen der Stiftung Michael in der November-Ausgabe (04/2013) der Zeitschrift für Epileptologie hingewiesen.

\section{Nicht vergessen - Frühbucher-Rabatt ....}

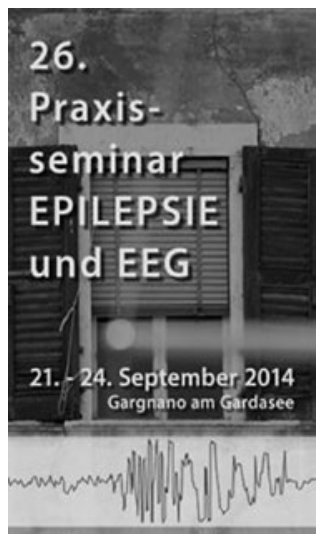

... für das Paxisseminar 2014 in Gargnano/Gardasee 